\title{
GIANT RENAL CYST MIMICKING ASCITES ON ABDOMINAL ULTRASONOGRAPHY
}

\author{
Martin Borovec ${ }^{1}$, Miroslav Solar̆ ${ }^{1}$, Jiři Ceral ${ }^{1}$, Antonín Michl ${ }^{2}$
}

Charles University in Prague, Faculty of Medicine and University Hospital Hradec Králové, Czech Republic: $1^{\text {st }}$ Department of Internal Medicine ${ }^{1}$, Department of Diagnostic Radiology ${ }^{2}$

Summary: An article describes diagnostic difficulties in patient with giant renal cyst, erroneously diagnosed as ascites on ultrasonographic examination. Patient was initially suspected to have disseminated intraabdominal malignancy. Abdominal paracentesis of supposed ascites was performed. The diagnosis of giant renal cyst was finally made by CT and patient was treated surgically. The limitations of ultrasonographic examination are pointed out are and a brief review of similar cases is given.

Key words: Renal cyst; Ascites; Ultrasonography

\section{Case report}

A 77-year-old patient was admitted to our department of internal medicine for two-weeks persisting anorexia, progressive weight loss, dehydration and indeterminate significant abdominal expansion. Patient was treated for coronary heart disease, arterial hypertension, hypercholesterolemia and chronic obstructive pulmonary disease. He was known to have asymptomatic bilateral renal-cortex cysts. His chronic medication consisted of ACE-inhibitor, fibrate, spironolacton, and slow release nitrate. Physical examination had revealed the signs of of hypohydration and mild subalimentation. Contact with patient was limited for bradypsychia. His blood pressure was $110 / 70 \mathrm{mmHg}$ and pulse was 78 per minute. Massive diffusive abdominal expansion in contrast with patient's asthenic habitus was the most evident pathology. The abdomen was painless on palpation and percussion, the abdominal wall was tough and distended. In laboratory examination, patient had normal blood count, slightly prolonged coagulation parameters, slightly elevated serum creatinine, he had normal levels of liver enzymes, normal glycaemia, total blood protein and albumin were not significantly reduced. We noted increased erythrocyte sedimentation rate. Chemical and microscopic urine analysis was normal. Voluminous ascites was described on ultrasonographic examination of abdomen, which was thought to be hemorrhagic due to higher echogenicity of the fluid and many septs present. Suspicion of tumourous infiltration of omentum was expressed. Biliary tract, liver parenchyma and gall bladder were normal, as well as right kidney (except a single cortex cyst with $3 \mathrm{~cm}$ diameter).
Due to voluminous presumable peritoneal fluid, left kidney and pancreas were not visualized and retroperitoneal area could not be examined.

We supposed patient to have a primary or secondary abdominal tumourous disease. In effort to confirm patient's diagnosis and to relieve from extensive ascites, we performed abdominal paracentesis and gained $1100 \mathrm{ml}$ of roily brownish fluid without any complications.

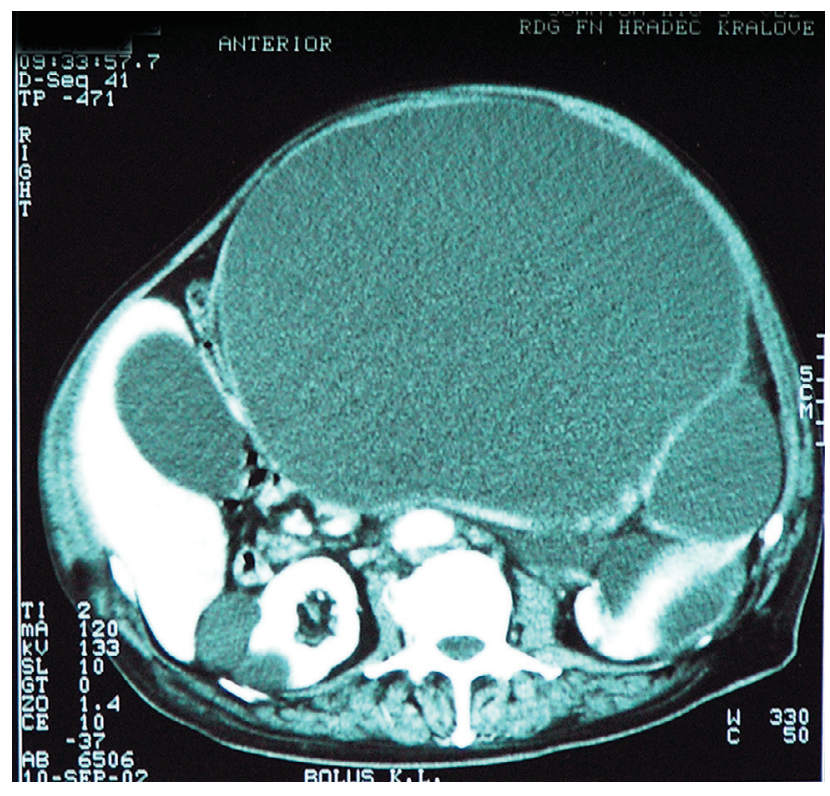

Fig. 1: CT scan of a giant renal cyst that is coming from the superior anterior aspect of the left kidney. 
Tab. 1: Other rare causes of cystic intraabdominal formations.

- Giant adrenal pseudocyst

- Gastrointestinal autonomic nerve sarcoma presenting as a giant intra-abdominal cyst

- Giant splenic epidermoid cyst

- Giant aneurysm of renal artery

- Giant gallbladder

- Giant sigmoidal diverticulum

- Giant abdominal pseudocyst resulting from ventriculoperitoneal shunt

- Giant intra-abdominal esophageal cyst
- Leiomyoma of the small intestine with giant cyst formation

- Acquired patent urachus and giant urachal cyst

- Giant cyst of the spermatic vesicle imitating abdominal tumor

- Giant abdominal lymphocele

- Giant choledochal cyst

- Giant congenital cysts of liver

- Lymphangiovenous malformation

- Giant cystic leiomyoma of the sigmoid colon
Laboratory examination of the fluid indicated transudate, which was negative in microbiological tests; TBC was excluded by PCR assay. No cythologic evidence of malignancy was identified.

We proceeded on performing CT examination of abdomen, which revealed a giant renal cyst with origin in left kidney, with $27 \mathrm{~cm}$ diameter (Fig. 1). There was no free fluid in abdominal cavity and no signs of intraabdominal malignancy were detected. The patient was referred for surgical treatment because of the expansive nature of the process. Although not the entire parenchyma of the left kidney was affected, left-sided nephrectomy was performed because of surgical reasons,. The removed renal tissue weighted 10 kilograms. There were no major complications of the surgery. Histological examination showed no signs of malignancy.

\section{Discussion}

For evaluation of abdominal pathologies, ultrasonography is a helpful examination. It is appreciated for its noninvasiveness, availability and no radiation stress. On the other hand, it is known for its operator-sensitivity. It still can give misguiding information, as shown in our case report.

Many authors described various diagnostic mistakes made by abdominal ultrasonography, misled by either fluidcontaining or solid intraabdominal formations. We focused on erroneously diagnosed ascites and noted a few cases, but none of them described any giant renal cyst imitating ascites. Nonpancreatic pseudocyst (4), giant ovarian cysts
(3), cysts of echinococcal (1) and omental (6) origin were misinterpreted as ascites. Giant renal cysts were mistaken for palpable gallbladder (5) and even for obesity (2). On the other hand, patient with a retroperitoneal venous aneurysm underwent surgery for diagnosis of giant left renal cyst (7). Cystic formations of vascular origin are rare, but in that case, Doppler ultrasound is a helpful modality. Other rare causes of inabdominal cystic formation are summarized in Tab. 1.

\section{Conclusion}

We conclude that the ultrasonography remains the first step exam in patients evaluated for intraabdominal pathology. However, it is necessary to be aware that in a minority of cases the ultrasonographic results may be misleading for clinicians.

\section{References}

1. Bar-Maor JA, Lernau OZ. Giant abdominal cysts simulating ascites. Am J Gastroenterol 1981;75:55-6.

2. Brown JA, Segura JW, Blute ML. A giant left renal cyst presenting as obesity: a unique presentation. Arch Esp Urol 1998;51:105-7.

3. Menahem S, Shvartzman P. Giant ovarian cyst mimicking ascites. J Fam Pract 1994;39:479-81.

4. Oray-Schrom P, St Martin D, Bartelloni P, Amoateng-Adjepong Y. Giant nonpancreatic pseudocyst causing acute anuria. J Clin Gastroenterol 2002;34:160-3.

5. Raghavendra BN, Ambos MA. Renal cyst masquerading as a palpable "gallbladder". JAMA 1981;246:125.

6. Rattan KN, Budhiraja S, Pandit SK, Yadav RK. Huge omental cyst mimicking ascites. Indian J Pediatr 1996;63:707-8.

7. Tsujimura A, Nishimura K, Matsumiya K, et al. A case of retroperitoneal venous aneurysm. Hinyokika Kiyo 1992;38:1037-40.

\section{Corresponding author:}

Miroslav Solař, M.D., Ph.D., $1^{\text {st }}$ Department of Internal Medicine, University Hospital Hradec Králové, Sokolská 581, Hradec Králové, 500 05, Czech Republic; e-mail: solarmir@seznam.cz 